\title{
Numerical investigation of localization and suppression of thermal decomposition of forest combustible materials using specialized water supply
}

\author{
Dmitrii Antonov ${ }^{1}$, Geniy Kuznetsov ${ }^{1}$, and Alena Zhdanova ${ }^{1, *}$ \\ ${ }^{1}$ National Research Tomsk Polytechnic University, 634050 Tomsk, Russia
}

\begin{abstract}
The danger of forest fires and their large-scale consequences are becoming ever more complex problems for mankind every year. The results of numerical studies of heat transfer processes under suppressing the thermal decomposition reaction of forest combustible materials by films and groups of water droplets are presented. We used forest combustible materials, typical for the Siberian region. We have established the relationship between the dimensions of the heated near-surface layer of the material and the thickness of the film layer of the liquid that evaporated when the thermal decomposition reaction was suppressed in forest combustible materials. Typical times of suppression of the thermal decomposition reaction of typical forest combustible materials are given when the water film on the surface evaporates. The influence of the mutual arrangement of droplets on the surface of the forest combustible material and the temperature of the outer vapor-gas mixture on the times of suppression of its pyrolysis is revealed. The results can be used to develop technologies for extinguishing fires, containment of flame sources and combustion front.
\end{abstract}

\section{Introduction}

Over the past five years, wildfires have claimed the lives of thousands of people around the world, the number of injured people exceeds tens of thousands [1-3]. In addition to thousands of hectares of burnt forest massifs, numerous cases of suffocation and poisoning of people with combustion products of forest combustible materials should be noted. The only possible way to solve the problem of forest fires is to create effective extinguishing technologies that will ensure the suppression of flame in the shortest possible time with the rational use of extinguishing agent [4].

Numerical [5] and experimental [6] studies have shown that in a typical local aviation discharge of water into the flame zone in the process of extinguishing forest fires, as a rule, only a small part (less than 10\%) of the liquid is used (directly evaporated). It was shown [7] that for a substantial (several hundred degrees) reduction in the flame temperature with

\footnotetext{
* Corresponding author: zhdanovaao@tpu.ru
} 
minimal water consumption, it is advisable to use a fine dispersion of the liquid. After the spraying time has elapsed, liquid films can be formed on the surface of the thermally decomposable material. It should be noted that the components of the forest massif (bark, leaves, and branches) can reach the surface of a burning forest material together with the liquid. In this case, an inhomogeneous system is formed on the surface of the thermally decomposable forest material, which includes water, leaves, needles, and bark. This system is a "buffer layer" between the surface of the thermally decomposable material and the environment. It should also be noted that only some of the droplets of the extinguishing liquid can reach the surface of the forest combustible material during movement through the flame [7]. In such a case, the formation of monolithic liquid films on the surface of the reacting material is unlikely with fine spraying (droplets fall at a certain distance between each other).

The purpose of this study is to numerically study the processes of heat transfer under conditions of endothermic phase transformations during the evaporation of a water film on the surface of typical forest combustible materials.

\section{Problem statement}

In the formulation of the heat transfer problem under consideration (Fig. $1 a$ ), it was assumed that the "water shell" (large set of water droplets) passed through the flame and the water was evenly distributed over the surface of the forest combustible material. A liquid film of fixed dimensions (thickness $L_{\mathrm{w}-\mathrm{f}}$ ) was formed. We assumed that the leaves, tree branches, bark, and needles together with the liquid reached the surface of the forest combustible material. Thus, a heterogeneous system (water with solid inclusions) is formed between a layer of forest combustible material with temperature $T \gg T_{\mathrm{d}}$ and a trace of a "water shell". Steam formation is intensified at the boundary of "forest combustible material - water with solid inclusions" when the phase transition conditions are reached. It was believed that the liquid was heated by heat exchange with the thermally decomposable forest material. At the boundary of the "water film - forest combustible material", vaporization occurs when the phase transition conditions are reached. The temperature of the forest combustible material is reduced due to the heat of the endothermic phase transition. During intensive vaporization, the thickness of the "water shell" (liquid film) decreases.

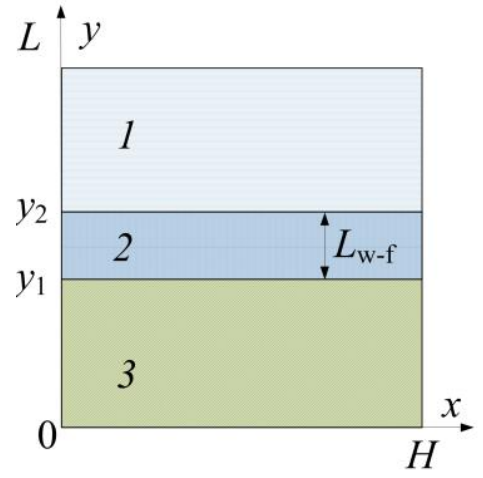

$a$

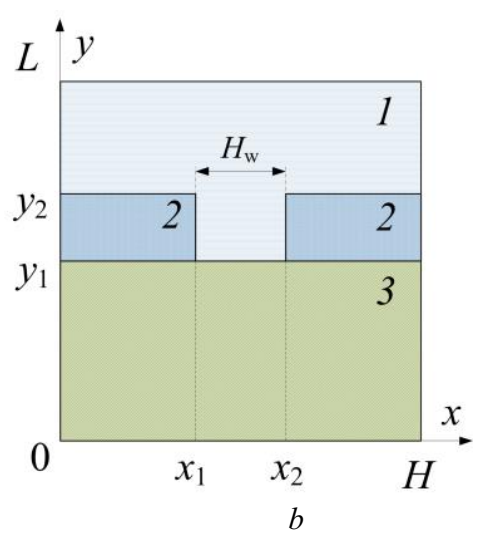

$b$

Fig. 1. Scheme of the solution domain for the heat transfer problem $\left(0<t<t_{\mathrm{d}}\right): a-$ upon evaporation of a water film with solid inclusions - ("buffer layer") on the surface of a forest combustible material; 1 - vapor-gas mixture, 2 - water, 3 - forest combustible material; $b$ - upon evaporation of water droplets on the surface of a forest combustible material; 1 - vapor-gas mixture, 2 - water droplets, 3 forest combustible material. 
In the formulation of the problem (Fig. $1 \mathrm{~b}$ ), it was assumed that a small group of drops had reached the surface of the thermally decomposable forest material. In general, different distances between individual water drops are possible on the surface of a forest combustible material under intense pyrolysis. From the whole variety of possible locations of the droplet group on the surface of the forest combustible material, the most significant for the subsequent analysis is the variant of two drops (Fig. $1 \mathrm{~b}$ ), located at a certain distance from each other $\left(H_{\mathrm{w}}=X_{2}-X_{1}\right)$. It was believed that the liquid is heated by heat exchange with a thermally decomposable forest material heated to high temperatures. Evaporation occurs at all boundaries of water droplets when the corresponding conditions are reached. The temperature of the forest combustible material is reduced due to the heat of the endothermic phase transition and conductive heat transfer.

Based on the results of numerical simulation, the time $\left(t_{\mathrm{d}}\right)$ of the decrease of the forest combustible material temperature to the temperature at which the thermal decomposition began $\left(T_{\mathrm{d}}\right)$. The value of $t_{\mathrm{d}}$ illustrates the time of suppression (termination) of the thermal decomposition reaction of a forest combustible material.

\section{Mathematical models and methods of solution}

When carrying out numerical studies, we used two software complexes - Ansys Fluent and Matlab. Mathematical statements for the systems (Fig. 1) are given in [8].

The problems were solved in the Matlab environment by nonstationary differential equations of heat transfer in partial derivatives for systems "vapor-gas mixture - water film with solid inclusions ("buffer layer") - forest combustible material" and "forest combustible material - water droplets - vapor-gas mixture" respectively. The system of nonstationary differential equations was solved by the method of finite differences [9]. The difference analogues of differential equations were solved by a locally one-dimensional method [10]. To solve one-dimensional difference equations, we used the sweep method using the implicit four-point scheme [9]. Nonlinear equations were solved by the iteration method [10]. Uneven time steps $\left(10^{-4} \div 10^{-2} \mathrm{~s}\right)$ and coordinate increments $\left(10^{-8} \div 10^{-6} \mathrm{~m}\right)$ were used to improve the accuracy of solving differential equations. Near the boundaries of the phase transition, the difference grid was condensed according to an algorithm similar to [5]. The method for evaluating the reliability of the theoretical studies results, based on the verification of the conservativeness of the difference scheme, is analogous to that used in [5].

When solving problems in Ansys Fluent, the finite element method was used. This method was originally included in this commercial software package. The solution of the heat and mass transfer problem assumed the developing a geometry of the tasks (Fig. 1), the formation of a grid area and a description of models of evaporation, combustion, thermal decomposition, turbulence, etc.

Numerical studies were performed with the following values of the parameters [9]: the initial temperature of water and gas-vapor mixture $T_{\mathrm{n}}=300 \mathrm{~K}$; the temperature of the onset of thermal decomposition of forest combustible material $T_{\mathrm{d}}=500 \mathrm{~K}$; the parameters of the chemical reaction were assumed to be identical for the considered types of forest combustible material $k_{3}{ }^{0}=3.63 \cdot 10^{4} \mathrm{~s}^{-1}, \quad E_{3}=78.114 \mathrm{~kJ} / \mathrm{mol}, \quad Q_{3}=1 \cdot 10^{3} \mathrm{~J} / \mathrm{kg}$; the dimensionless coefficient of condensation (evaporation) $\beta=0.1$; molar mass of water $M=18$ $\mathrm{kg} / \mathrm{kmol}$; thermal effect of water evaporation $Q_{\mathrm{e}}=2.26 \mathrm{MJ} / \mathrm{kg}$. The thickness of the nearsurface layer of forest combustible material was varied in the range $L_{\mathrm{f}}=0.02-0.06 \mathrm{~mm}$. The thickness of the liquid film was assumed equal to $L_{\mathrm{w}}=0.01 \mathrm{~mm}$. The size of the solution area was changed depending on the values of $L_{\mathrm{f}}$ in the range $L=0.02-0.2 \mathrm{~m}$. The longitudinal and transverse dimensions of the droplets were varied in the range $H_{\mathrm{dr}}=0.01-$ $0.05 \mathrm{~m}$ at $L_{\mathrm{dr}}=0.01 \mathrm{~m}$. The distance between droplets was varied in the range $H_{\mathrm{w}}=0.001-$ 
$0.09 \mathrm{~m}$. The characteristic dimensions of the solution region were varied depending on the values of $H_{\mathrm{dr}}, H_{\mathrm{w}}$ and $L_{\mathrm{f}}$ in the ranges $H=0.05-0.1 \mathrm{~m}, L=0.05-0.1 \mathrm{~m}$. Thermophysical characteristics of forest combustible materials (birch leaves, pine needles and spruce needles), of pyrolysis products, of water and water vapor were taken according to $[11,12]$.

\section{Results and Discussion}

Fig. 2 shows the characteristic times of suppression of the thermal decomposition of forest combustible material under the impact of a water film with solid inclusions. It was found that the inertia of the process of suppressing the thermal decomposition reaction increases substantially with changing $L_{\mathrm{w}-\mathrm{f}}$ in the range from $0.002 \mathrm{~m}$ to $0.004 \mathrm{~m}$. With further growth of $L_{\mathrm{w}-\mathrm{f}}$ the times $t_{\mathrm{d}}$ almost do not change (deviations less than 1\%). This result can be explained by the small mass of water evaporating upon suppression of the decomposition reaction.

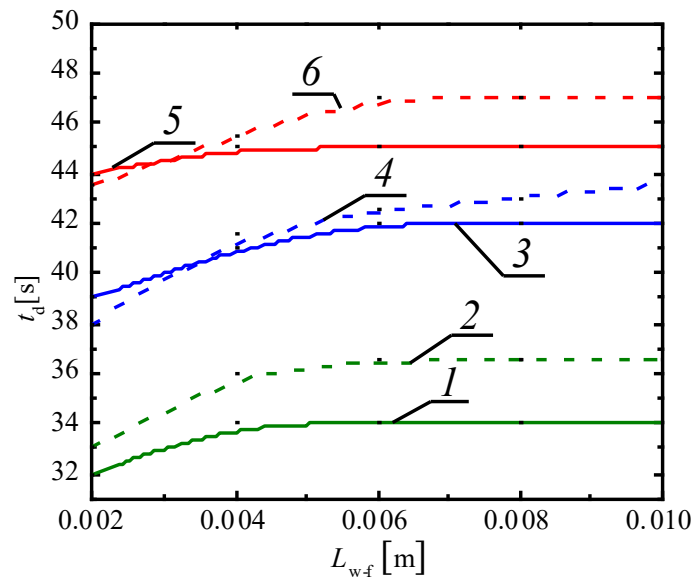

Fig. 2. Characteristic times of suppression of the thermal decomposition reaction of the forest combustible material depending on the thickness of the water film $L_{\mathrm{w}-\mathrm{f}:} 1,3,5$ - results of numerical studies in Matlab; 2, 4, 6- results of numerical studies in Ansys Fluent; 1, 2- pine needles, 3, 4birch leaves, 5, 6 - spruce needles.

We have established that the considered "buffer layer" is heated to a depth of less than $0.005 \mathrm{~m}$ and the thickness of the evaporated water layer is about $0.001 \mathrm{~m}$. Therefore, the possible increase of $L_{\mathrm{w}-\mathrm{f}}$ relatively to $0.005 \mathrm{~m}$ almost does not affect the cooling conditions of the forest material and, as a consequence, the conditions for suppressing the reaction of its decomposition. Fig. 3 illustrates the dependence of the times of suppression of the thermal decomposition of forest combustible material on the distance between two water droplets $\left(H_{\mathrm{w}}\right)$ at the temperatures in the trace of the "water shell" 300-350 K. It can be noted that with decreasing $H_{\mathrm{w}}$, the times of the thermal decomposition reaction termination $t_{\mathrm{d}}$ are decrease nonlinearly. 


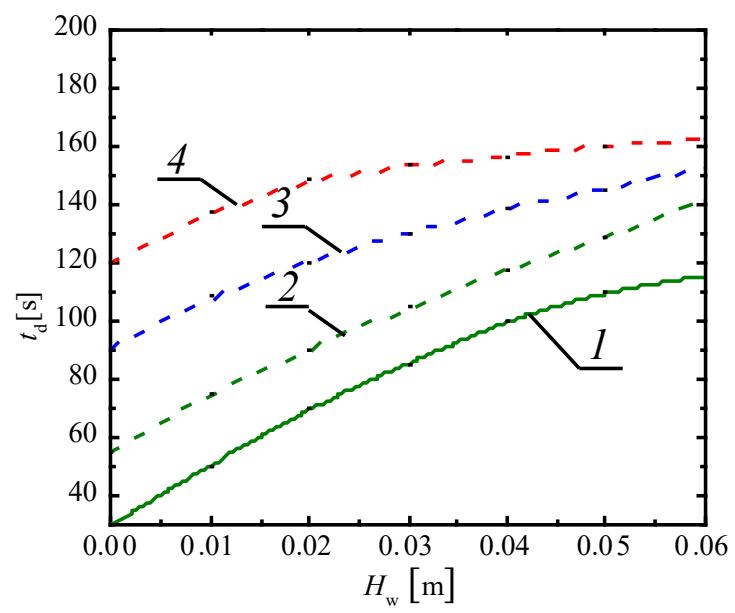

Fig. 3. Characteristic times of suppression of the thermal decomposition reaction of the forest combustible material depending on the distance between water droplets $H_{\mathrm{w}}$ when extinguishing by single water droplets: 1 - results of numerical studies in Matlab; $2-4$-results of numerical studies in Ansys Fluent; 1,2 - pine needles, 3 - birch leaves, 4 - spruce needles.

The established (Fig. 3) dependences $t_{\mathrm{d}}=f\left(H_{\mathrm{w}}\right)$ can be explained by the fact that the temperature of the vapor-gas mixture in the region between the droplets increases significantly under the thermal decomposition of the forest material due to intense heat release. A large proportion of this heat is consumed for the implementation of phase transformations on the lateral boundaries of droplets $\left(X=X_{1}, X=X_{2}, Y_{1}<Y<Y_{2}\right)$ when the distances between the droplets is less than $0.02 \mathrm{~m}$ (due to the large thermal effect of liquid evaporation, $Q_{2}=2.26 \mathrm{MJ} / \mathrm{kg}$ ). In this case, the area of heating of the vapor-gas mixture is limited by the thickness of the liquid droplets $L_{\mathrm{dr}}$ (Fig. 1). A significant increase in the size of the heating region of the vapor-gas mixture relatively to $L_{\mathrm{dr}}$ was established (Fig. 1) with increasing the values of $H_{\mathrm{w}}$.

Deviations of the numerical studies results in Matlab and Ansys Fluent are primarily due to the fact that the rate of water evaporation is given by a constant in Ansys Fluent, but is determined by the experimental approximations, if Matlab is used.

\section{Conclusion}

Excessive flooding of the surface of forest combustible material with water is wasteful to reduce the possibility of its thermal decomposition. A special spraying of the liquid with the formation of a small amount of water on the forest combustible material surface is recommended $\left(L_{\mathrm{w}-\mathrm{f}}=1-3 \mathrm{~mm}\right)$. Even in the presence of "buffer layers" (water films with solid inclusions) considered in this work, it is possible to stop forest material pyrolysis in sufficiently short (less than 2 minutes) times.

However, with specialized spraying, the probability of forming relatively large "buffer layers" is significantly reduced due to low water consumption and a small fraction of leaves, branches and bark carried away by the liquid.

Numerical studies have shown that the distance between water droplets $\left(H_{\mathrm{w}}\right)$ significantly affects the termination of the reaction of thermal decomposition of forest combustible material (birch leaves, pine needles and spruce). It was shown that the times $t_{\mathrm{d}}$ can differ by $10-40 \%$ for identical values of $H_{\mathrm{w}}$ as a function of the temperature of the vapor-gas mixture at the surface of the forest combustible material and, consequently, between the droplets of the liquid. 
The research was funded by the Russian Science Foundation (project 18-19-00056).

\section{References}

1. A. Dimitrakopoulos, C. Gogi, G. Stamatelos, I. Mitsopoulos, Pol. J. Environ. Stud. 20, 327 (2011)

2. F. X. Catry, F. C. Rego, F. Moreira, F. Bacao, 1st International Conference on Modelling, Monitoring and Management of Forest Fires 119, 213 (2008), DOI: 10.2495/FIVA080221

3. D. H. Klyde, D. J. Alvarez, P. C. Schulze, T. H. Cox, M. Dickerson, AIAA Atmospheric Flight Mechanics Conference 97625 (2010)

4. A. O. Zhdanova, R. S. Volkov, I. S. Voytkov, K. Y. Osipov, G. V. Kuznetsov, Int. J. Heat Mass Transfer 126, 703 (2018), DOI: 10.1016/j.ijheatmasstransfer.2018.05.085

5. R. S. Volkov, G. V. Kuznetsov, P. A. Strizhak, Int. J. Heat Mass Transfer 79, 838 (2014), DOI: 10.1016/j.jiheatmasstransfer.2014.09.006

6. R. S. Volkov, G. V. Kuznetsov, P. A. Strizhak, Int. J. Heat Mass Transfer 96, 20 (2016), DOI: 10.1016/j.ijheatmasstransfer.2016.01.029

7. R. S. Volkov, G. V. Kuznetsov, P. A. Strizhak, Int. J. Heat Mass Transfer 85, 1 (2015), DOI: 10.1016/j.ijheatmasstransfer.2015.01.078

8. A. O. Zhdanova, G. V. Kuznetsov, J. C. Legros, P. A. Strizhak. Therm. Sci. 21, 2565 (2017), DOI: 10.2298/TSCI151006121Z

9. A. A. Samarskij, Teorija raznostnyh shem [The theory of difference schemes] (Nauka, Moskow, 1983) [in Russian]

10. L. A. Kozdoba, Metody reshenija nelinejnyh zadach teploprovodnosti [Methods for solving nonlinear heat conduction problems] (Nauka, Moskow, 1975) [in Russian]

11. N. B. Vargaftik, Spravochnik po teplofizicheskim svoistvam gazov i zhidkostey [Gases and liquids thermophysical properties guide] (Stars, Moskow, 2006) [in Russian]

12. V. N .Yurenev, P. D. Lebedev, Teplotechnicheskiy spravochnik [Thermophysical guide] (Energiia, Moskow, 1975) [in Russian] 